\title{
Algebraic independence of the values of Mahler functions satisfying implicit functional equations
}

\author{
by \\ Bernd Greuel (Köln)
}

1. Introduction and results. In a sequence of three papers Mahler ([4]-[6]) discussed the transcendence and algebraic independence of values of functions in several variables satisfying a certain type of functional equation. In his survey article [7], 37 years later, he stated three new problems. The third problem (for the first and second problem cf. Loxton and van der Poorten [3]) dealt with implicit functional equations of the type

$$
P(z, f(z), f(T z))=0
$$

with $T z=z^{d}, d \in \mathbb{Z}, d \geq 2$ and a polynomial $P(z, y, u)$ with coefficients in $\overline{\mathbb{Q}}$, the algebraic closure of $\mathbb{Q}$. Nishioka [8] (cf. Chapter 1.5 in [11]) solved this problem for polynomial transformations $T$. In [9] she extended her method to functions in several variables and suitable generalizations of the transformation $T z=z^{d}$.

Becker [1] generalized the result of Nishioka to algebraic transformations $T$. Töpfer gave in [15] a quantitative version of Becker's result. In that article Töpfer asked for a proof of the algebraic independence of the values of several functions satisfying implicit functional equations at algebraic points.

In this paper we follow the proof of Töpfer [15] and derive a lower bound for the transcendence degree of the values of functions $f_{1}, \ldots, f_{m}$ satisfying a special system of implicit functional equations for the transformation $T z=z^{d}$ with an integer $d \geq 2$. It should be easy to generalize the following result to polynomial or even rational or algebraic transformations $T$ (cf. Becker [1] and Töpfer [14, 15]).

For the development of Mahler's method in the last 15 years see the monograph of Nishioka [11] and the overview article of Waldschmidt [16] for further references.

2000 Mathematics Subject Classification: 11J82, 11J91. 
Throughout the paper let $\mathbb{K}$ denote an algebraic number field and $\mathcal{O}_{\mathbb{K}}$ the ring of integers in $\mathbb{K}$. As usual we denote by $\mid \alpha$ the house of an algebraic number $\alpha$, which is the maximum of the absolute values of the conjugates of $\alpha$. A denominator of an algebraic number $\alpha$ is a positive integer $D$ such that $D \alpha \in \mathcal{O}_{\mathbb{K}}$. If $P\left(z, y_{1}, \ldots, y_{m}\right)=: P(z, y)$ is a polynomial with complex coefficients, $\operatorname{deg}_{z} P=: d_{z} P$ denotes the partial degree of $P$ with respect to $z$, $\operatorname{deg}_{y} P=: d_{\underline{y}} P$ denotes the total degree in $\underline{y}:=\left(y_{1}, \ldots, y_{m}\right)$ and analogous notations in other cases. If the coefficients of $P$ are algebraic, the height $H(P)$ of $P$ is defined as the maximum of the houses of the coefficients of $P$, and the length $L(P)$ is the sum of the houses of the coefficients. In what follows let $c, c_{0}, c_{1}, \ldots$ and $\gamma_{0}, \gamma_{1}, \ldots$ denote positive constants which are independent of the parameters $M, N, k, k_{0}, k_{1}$ used. For a vector $\mu \in \mathbb{C}^{m}$ we define $|\mu|:=\left|\mu_{1}\right|+\ldots+\left|\mu_{m}\right|$ and by $\mathbb{N}$ and $\mathbb{N}_{0}$ we denote the positive and nonnegative integers.

TheOREM 1. Let $f_{1}, \ldots, f_{m}$ be analytic in a neighborhood $U$ of the origin, algebraically independent over $\mathbb{C}(z)$ and suppose that the coefficients of their power series

$$
f_{i}(z)=\sum_{j=0}^{\infty} f_{i, j} z^{j} \quad(i=1, \ldots, m)
$$

belong to a fixed algebraic number field $\mathbb{K}$ and satisfy

$$
\left|f_{i, j}\right| \leq \exp \left(c_{0}\left(1+j^{L}\right)\right) \quad \text { and } \quad D^{\left[c_{0}\left(1+j^{L}\right)\right]} f_{i, j} \in \mathcal{O}_{\mathbb{K}}
$$

for $j \in \mathbb{N}_{0}$ and $i=1, \ldots, m$ with suitable constants $D \in \mathbb{N}$ and $L \geq 1$. Let $\underline{n} \in \mathbb{N}^{m}$ and $\beta:=n_{1} \cdot \ldots \cdot n_{m}$. Suppose that the functions $f_{1}, \ldots, f_{m}$ satisfy the functional equations

$$
a(z) f_{j}\left(z^{d}\right)^{n_{j}}=\sum_{\nu=0}^{n_{j}-1} P_{\nu, j}(z, \underline{f}(z)) f_{j}\left(z^{d}\right)^{\nu}
$$

with polynomials $a \in \overline{\mathbb{Q}}[z] \backslash\{0\}$ and $P_{0,1}, \ldots, P_{n_{m}-1, m} \in \overline{\mathbb{Q}}[z, y]$ and an integer d satisfying $d>\max \left\{\beta^{L}, d_{\underline{y}}(\underline{P})\right\}$, where $d_{\underline{y}}(\underline{P})$ is defined by

$$
d_{\underline{y}}(\underline{P}):=\max \left\{\operatorname{deg}_{\underline{y}}\left(P_{0,1}\right), \ldots, \operatorname{deg}_{\underline{y}}\left(P_{n_{m}-1, m}\right)\right\} .
$$

Assume $\alpha \in \overline{\mathbb{Q}}^{*} \cap U$ and $a\left(\alpha^{d^{k}}\right) \neq 0$ for all $k \in \mathbb{N}_{0}$. Let $m_{0}$ be the smallest integer satisfying

$$
m_{0} \geq \frac{m \log d-L(m+1) \log \beta\left(1+\frac{\log \beta}{\log d}\right)}{\log \beta+\log d+\left(L(m+1)\left(1+\frac{\log \beta}{\log d}\right)+m\right)\left(2 \log \beta+\log d_{\underline{y}}(\underline{P})\right)} .
$$

Then

$$
\operatorname{trdeg}_{\mathbb{Q}} \mathbb{Q}\left(f_{1}(\alpha), \ldots, f_{m}(\alpha)\right) \geq m_{0}
$$


As an application of this theorem we obtain easily the following

Corollary 2. Under the assumptions of Theorem 1 , if $\alpha, f$ and the parameters $d, \beta$ and $d_{\underline{y}}(\underline{P})$ satisfy for $m>1$ the inequality

$$
\frac{\log d_{\underline{y}}(\underline{P})}{\log d}<\frac{1-\frac{\log \beta}{\log d}\left(2 m^{2}-m-1+L(m+1)\left(1+\frac{\log \beta}{\log d}\right)(2 m-1)\right)}{(m-1)\left(L(m+1)\left(1+\frac{\log \beta}{\log d}\right)+m\right)},
$$

then $f_{1}(\alpha), \ldots, f_{m}(\alpha)$ are algebraically independent.

REMARKs. (i) Nishioka [8] proved the transcendence of $f(\alpha)$ under the condition $d^{2}>n^{2} \max \left\{d, \operatorname{deg}_{y}(P)\right\}$, where $f$ satisfies the functional equation (1) and $n=\operatorname{deg}_{u}(P)$.

Under the hypotheses of Theorem 1 we get the transcendence of $f(\alpha)$ only under the stronger condition $d>\max \left\{n^{\sqrt{3}+1}, \operatorname{deg}_{y}(P)\right\}$. The reason for this is that we have to construct a sequence of polynomials $\left(Q_{k}\right)_{k_{0} \leq k \leq k_{1}}$, where the difference $k_{1}-k_{0}$ has to be relatively large (cf. Lemma 8). In the simpler case $m=1$ it suffices to find just one integer $k$ to obtain a contradiction. By an improvement of the method of proof we get the transcendence of $f(\alpha)$ under the condition $d>\max \left\{n^{2}, \operatorname{deg}_{y}(P)\right\}$, which coincides with the condition of Nishioka in the case $d>\operatorname{deg}_{y}(P)$. Note that we have to assume $d>d_{y}(\underline{P})$ only for technical reasons (cf. formula (24)).

(ii) Töpfer proved in [15] a transcendence measure for $f(\alpha)$ under the condition $d>n \max \left\{n, \operatorname{deg}_{y}(P)\right\}$.

(iii) For $m \geq 1$ and $\beta=1$ we get the result of Nishioka [10]. In [10] one can also find a lot of applications. For other examples in this case, but $d_{\underline{y}}(\underline{P})=1$, see Chirskiı [2] and Töpfer [14].

Our next example deals with infinite products of the form

$$
f_{n}(z):=\prod_{j=0}^{\infty}\left(1-z^{d^{j}}\right)^{n^{j}}
$$

where $d$ and $n$ are positive integers with $d \geq 2$.

Let $1 \leq n_{1}<\ldots<n_{m}(m \geq 2)$. Then the functions $f_{n_{i}}$ are analytic for $|z|<1$ and satisfy the functional equations

$$
f_{n_{i}}(z)=(1-z) f_{n_{i}}\left(z^{d}\right)^{n_{i}} \quad(i=1, \ldots, m) .
$$

Hence we have the following:

Corollary 3. Let $1 \leq n_{1}<\ldots<n_{m}$ be integers and $\beta:=n_{1} \cdot \ldots \cdot n_{m}$. If $\alpha$ is algebraic with $0<|\alpha|<1$ and $d$ is an integer with

$$
\log d>\left(2 m^{2}-1+\sqrt{4 m^{4}-2 m^{2}+m}\right) \log \beta,
$$


then the values

$$
\prod_{j=0}^{\infty}\left(1-\alpha^{d^{j}}\right)^{n_{1}^{j}}, \ldots, \prod_{j=0}^{\infty}\left(1-\alpha^{d^{j}}\right)^{n_{m}^{j}}
$$

are algebraically independent over $\mathbb{Q}$. Under the corresponding conditions on $\alpha, d$ and $n$ we get the algebraic independence of

$$
\prod_{j=0}^{\infty}\left(1-\alpha^{d^{j}}\right), \prod_{j=0}^{\infty}\left(1-\alpha^{d^{j}}\right)^{2^{j}}, \ldots, \prod_{j=0}^{\infty}\left(1-\alpha^{d^{j}}\right)^{n^{j}} .
$$

REMARK. Nishioka proved (Theorem 3.4.13 in [11]) the algebraic independence of

$$
\prod_{j=0}^{\infty}\left(1-\alpha^{d^{j}}\right) \quad(d=2,3, \ldots)
$$

for any algebraic number $\alpha$ with $0<|\alpha|<1$.

Proof (of Corollary 3). The algebraic independence of the functions $f_{n_{1}}, \ldots, f_{n_{m}}$ over $\mathbb{C}(z)$ will be shown in the last section.

By the remark after Lemma $4, f_{n_{1}}, \ldots, f_{n_{m}}$ satisfy the conditions for the houses and denominators of the coefficients in Theorem 1 for any $L>1$. Then the assumption of Corollary 3 follows immediately from Theorem 1 and Corollary 2.

2. Preliminaries and auxiliary results. For $\mu \in \mathbb{N}_{0}, \mu \in \mathbb{N}_{0}^{m}$ and $f_{i}(z):=\sum_{j=0}^{\infty} f_{i, j} z^{j}(i=1, \ldots, m)$ we define

$(4)$

$$
\begin{gathered}
f_{i}(z)^{\mu}:=\sum_{j=0}^{\infty} f_{i, j}^{(\mu)} z^{j}, \quad f_{i, j}^{(\mu)}:=\sum_{\substack{\nu_{1}, \ldots, \nu_{\mu} \in \mathbb{N}_{0} \\
\nu_{1}+\ldots+\nu_{\mu}=j}} f_{i, \nu_{1}} \cdot \ldots \cdot f_{i, \nu_{\mu}}, \\
\underline{f}(z)^{\underline{\mu}}:=f_{1}(z)^{\mu_{1}} \cdot \ldots \cdot f_{m}(z)^{\mu_{m}}=\sum_{j=0}^{\infty} f_{j}^{(\underline{\mu})} z^{j}, \\
f_{j}^{(\underline{\mu})}:=\sum_{\substack{\nu_{1}, \ldots, \nu_{m} \in \mathbb{N}_{0} \\
\nu_{1}+\ldots+\nu_{m}=j}} f_{1, \nu_{1}}^{\left(\mu_{1}\right)} \cdot \ldots \cdot f_{m, \nu_{m}}^{\left(\mu_{m}\right)} .
\end{gathered}
$$

Lemma 4. If $f_{i, j} \leq \exp \left(c_{0}\left(1+j^{L}\right)\right)$ and $D^{\left[c_{0}\left(1+j^{L}\right)\right]} f_{i, j} \in \mathcal{O}_{\mathbb{K}}$ for $i=$ $1, \ldots, m$ and all $j \in \mathbb{N}_{0}$ with $L \geq 1$ and $D \in \mathbb{N}$, then for all $\mu \in \mathbb{N}_{0}$ and $\underline{\mu} \in \mathbb{N}_{0}^{m}$ the following assertions hold:

(i) $\left|f_{i, j}^{(\mu)}\right| \leq \exp \left(c_{1}\left(\mu+j^{L}\right)\right), D^{\left[c_{1}\left(\mu+j^{L}\right)\right]} f_{i, j}^{(\mu)} \in \mathcal{O}_{\mathbb{K}}$,

(ii) $\mid f_{j}^{(\underline{\mu})} \leq \exp \left(c_{2}\left(|\underline{\mu}|+j^{L}\right)\right), D^{\left[c_{2}\left(|\underline{\mu}|+j^{L}\right)\right]} f_{j}^{(\underline{\mu})} \in \mathcal{O}_{\mathbb{K}}$. 
Proof. Assertions (i) and (ii) are consequences of the identities (3) and (4) using the fact that the number of $\underline{\nu} \in \mathbb{N}_{0}^{\mu}$ with $\nu_{1}+\ldots+\nu_{\mu}=j$ is bounded by $\left(\begin{array}{c}j+\mu-1 \\ \mu-1\end{array}\right) \leq 2^{j+\mu}$.

REMARK. If the functions $f_{1}, \ldots, f_{m}$ satisfy functional equations of type

$$
P_{i}\left(z, f_{i}(z), f_{i}\left(z^{d}\right)\right)=0 \quad(i=1, \ldots, m)
$$

with polynomials $P_{i} \in \overline{\mathbb{Q}}[z, y, u] \backslash\{0\}$ and $\operatorname{deg}_{u}\left(P_{i}\right) \geq 1$, we see that there exist an algebraic number field $\mathbb{K}$, an explicit computable constant $c>0$ and a positive integer $D \in \mathbb{N}$ such that for $j \in \mathbb{N}_{0}$ and all $\varepsilon>0$ :

(i) $f_{i, j} \in \mathbb{K}$

(ii) $\left\lceil f_{i, j} \leq \exp \left(c\left(1+j^{1+\varepsilon}\right)\right)\right.$,

(iii) $D^{1+j} f_{i, j} \in \mathcal{O}_{\mathbb{K}}$

hold, i.e. the conditions of Lemma 4 are fulfilled for all $L>1$. For a proof of this remark see Lemma 1.5.3 of Nishioka [11] and Proposition 1 of Becker [1] for a more general result.

Lemma 5. For $N \in \mathbb{N}$ there exists a polynomial $R \in \mathcal{O}_{\mathbb{K}}[z, y] \backslash\{0\}$ with the following properties:

(i) $\operatorname{deg}_{z} R \leq N, \operatorname{deg}_{y} R \leq N$,

(ii) $\log H(R) \leq c_{3} N^{(m+1) L}$,

(iii) $\nu:=\operatorname{ord}_{0} R(z, \underline{f}(z)) \geq c_{4} N^{m+1}$

for suitable constants $c_{3}, c_{4} \in \mathbb{R}_{+}$.

Proof. Put

$$
R(z, \underline{y}):=\sum_{\lambda=0}^{N} \sum_{|\underline{\mu}| \leq N} r_{\lambda, \underline{\mu}} z^{\lambda} \underline{y^{\mu}}
$$

with $(N+1)\left(\begin{array}{c}N+m \\ m\end{array}\right)$ unknown coefficients $r_{\lambda, \underline{\mu}}$. Then

$$
R(z, \underline{f}(z)):=\sum_{\lambda=0}^{N} \sum_{|\underline{\mu}| \leq N} r_{\lambda, \underline{\mu}} z^{\lambda} \underline{f}(z) \underline{\mu}=\sum_{h=0}^{\infty} \beta_{h} z^{h} \quad \text { (say) }
$$

with (cf. the identity (4))

$$
\beta_{h}=\sum_{\lambda=0}^{\min \{h, N\}} \sum_{|\underline{\mu}| \leq N} r_{\lambda, \underline{\mu}} f_{h-\lambda}^{(\underline{\mu})} .
$$

Assertion (iii) is equivalent to the condition $\beta_{h}=0$ for $0 \leq h<c_{4} N^{m+1}$, and this yields at most $\left[c_{4} N^{m+1}\right]+1$ equations in the

$$
(N+1)\left(\begin{array}{c}
N+m \\
m
\end{array}\right) \geq \frac{1}{m !} N^{1+m}>2 c_{4} N^{m+1}+1
$$


unknowns $r_{\lambda, \underline{\mu}}$ for a suitable constant $c_{4}$. After multiplication with a suitable denominator $D^{\left[c_{2} N^{(1+m) L}\right]}$ according to Lemma 4 the coefficients $f_{h-\lambda}^{(\underline{\mu})}$ are algebraic integers and their houses are bounded by $\exp \left(c_{5}\left(N^{(1+m) L}\right)\right)$. Siegel's lemma (cf. Hilfssatz 31 in Schneider [12]) yields the assertion.

Lemma 6. Let $\nu$ be as in Lemma 5 and $\beta_{h}$ denote the Taylor coefficients of $R(z, \underline{f}(z))$ as in the proof. Then

(i) $\left|\beta_{h}\right| \leq \exp \left(c_{6}\left(h+N^{(1+m) L}\right)\right) \leq \exp \left(c_{7}\left(h+\nu^{L}\right)\right)$.

(ii) $\left|\beta_{\nu}\right| \geq \exp \left(-c_{8} \nu^{L}\right)$.

(iii) Suppose that $k \in \mathbb{N}$ satisfies $d^{k} \geq c_{9} \nu^{L}$ with $\nu, N, L$ as above and a suitable constant $c_{9} \in \mathbb{R}_{+}$depending only on $f$ and $\alpha$. Then there exist constants $c_{10}, c_{11} \in \mathbb{R}_{+}$depending only on $\underline{f}$ and $\alpha$ such that

$$
-c_{10} \nu d^{k} \leq \log \left|R\left(T^{k}(\alpha), \underline{f}\left(T^{k}(\alpha)\right)\right)\right| \leq-c_{11} \nu d^{k},
$$

where $T^{k}(\alpha)$ denotes the $k$ th iterate of $T$ at the point $\alpha$.

Pro of. From (5) we get

$$
\beta_{h}=\sum_{\lambda=0}^{\min \{h, N\}} \sum_{|\underline{\mu}| \leq N} r_{\lambda, \underline{\mu}} f_{h-\lambda}^{(\underline{\mu})} .
$$

This representation together with Lemma 5 and the inequality $\left|f_{i, j}\right| \leq$ $\exp \left(\gamma_{0}(j+1)\right)$ (notice that the functions $f_{1}, \ldots, f_{m}$ are analytic in a neighborhood of 0$)$, hence $\left|f_{h}^{(\underline{\mu})}\right| \leq \exp \left(\gamma_{1}(|\underline{\mu}|+h)\right)$ with $\gamma_{0}, \gamma_{1} \in \mathbb{R}_{+}$, implies the first estimate of Lemma 6.

For $D, L, c_{4}$ as above and $\nu$ as in Lemma 5 we get (recall $\nu \geq c_{4} N^{1+m}$ )

$$
D^{\left[\gamma_{2}\left(N+\nu^{L}\right)\right]} \beta_{\nu} \in \mathcal{O}_{\mathbb{K}}
$$

and

$$
\left|\beta_{\nu}\right| \leq \exp \left(\gamma_{3}\left(N^{(1+m) L}+\nu^{L}+N\right)\right) \leq \exp \left(\gamma_{4} \nu^{L}\right) .
$$

By a Liouville estimate we obtain the second part.

We now come to the last part of Lemma 6 . By Lemma 5 we write

$$
R\left(T^{k}(\alpha), \underline{f}\left(T^{k}(\alpha)\right)\right)=\beta_{\nu}\left(T^{k}(\alpha)\right)^{\nu}\left(1+\sum_{h=1}^{\infty} \frac{\beta_{h+\nu}}{\beta_{\nu}}\left(T^{k}(\alpha)\right)^{h}\right)
$$

and by the assumption on $k$ and the first two parts of Lemma 6 we get

$$
\begin{aligned}
\left|\sum_{h=1}^{\infty} \frac{\beta_{h+\nu}}{\beta_{\nu}}\left(T^{k}(\alpha)\right)^{h}\right| & \leq \sum_{h=1}^{\infty} \exp \left(c_{7}\left(\nu^{L}+h\right)+c_{8} \nu^{L}-\gamma_{5} h d^{k}\right) \\
& \leq \sum_{h=1}^{\infty} \exp \left(\gamma_{6} \nu^{L}-\gamma_{7} h d^{k}\right)<\frac{1}{2}
\end{aligned}
$$


Now the assertion follows from $\left|T^{k}(\alpha)\right|^{\nu}=\exp \left(-\gamma_{8} \nu d^{k}\right)$ and $\exp \left(-c_{8} \nu^{L}\right) \leq$ $\left|\beta_{\nu}\right| \leq \exp \left(2 c_{7} \nu^{L}\right)$.

Lemma 7. Let $S, U_{1}, \ldots, U_{d} \in \mathbb{C}$ satisfy $S^{d}+U_{1} S^{d-1}+\ldots+U_{d}=0$ and

$$
-X_{1} \leq \log |S| \leq-X_{2}, \quad \log \left|U_{i}\right| \leq Y \quad(1 \leq i \leq d)
$$

for $X_{1}, X_{2}, Y \in \mathbb{R}_{+}$. Then there exists $j \in\{1, \ldots, d\}$ such that

$$
-d X_{1}-Y-\log d \leq \log \left|U_{j}\right| \leq-X_{2}+Y+\log d .
$$

Proof. This is Lemma 4.2.3 of Wass [17].

REMARK. The examples $S^{d}+U_{d}=0$ and $S^{d}+U_{1} S^{d-1}=0$ show that the bounds for $\left|U_{j}\right|$ cannot be improved.

The proof of Theorem 1 depends on the following result from elimination theory, which can be found in Töpfer [13, Theorem 1] with slight modifications.

Lemma 8. Suppose $\underline{\omega} \in \mathbb{C}^{m}$ and $\mathbb{K}$ is an algebraic number field. Then there exists a constant $c_{12}=c_{12}(\omega, \mathbb{K}) \in \mathbb{R}_{+}$with the following property: If there exist increasing functions $\psi_{1}, \psi_{2}, \Lambda: \mathbb{N} \rightarrow \mathbb{R}_{+}$, real numbers $\Phi_{2} \geq \Phi_{1} \geq c_{12}$, positive integers $k_{0}<k_{1}, m_{0} \in\{0, \ldots, m\}$ and polynomials $\left(Q_{k}\right)_{k_{0} \leq k \leq k_{1}} \in \mathcal{O}_{\mathbb{K}}[\underline{y}]$ such that the following assumptions are satisfied:

(i) $1 \leq \psi_{1}(k+1) / \psi_{2}(k) \leq \Lambda(k)$ and $\psi_{2}(k) \geq c_{12}\left(\log H\left(Q_{k}\right)+\operatorname{deg}_{y} Q_{k}\right)$ for $k \in\left\{k_{0}, \ldots, k_{1}\right\}$,

(ii) the polynomials $\left(Q_{k}\right)_{k_{0} \leq k \leq k_{1}}$ satisfy, for $k \in\left\{k_{0}, \ldots, k_{1}\right\}$,
(a) $\operatorname{deg}_{y} Q_{k} \leq \Phi_{1}$
(b) $\log H\left(Q_{k}\right) \leq \Phi_{2}$,
(c) $-\psi_{1}(k) \leq \log \left|Q_{k}(\underline{\omega})\right| \leq-\psi_{2}(k)$,

(iii) $\psi_{2}\left(k_{1}\right) \geq c_{12} \Lambda\left(k_{1}\right)^{m_{0}-1} \Phi_{1}^{m_{0}-1} \max \left\{\psi_{1}\left(k_{0}\right), \Phi_{2}\right\}$,

then $\operatorname{trdeg} \operatorname{de}_{\mathbb{Q}} \mathbb{Q}(\underline{\omega}) \geq m_{0}$.

3. Construction of an auxiliary function. Since the case $\beta=1$ (i.e. $n_{1}=\ldots=n_{m}=1$ ) was treated by Nishioka [10] we can assume $\beta>1$.

The proof is rather long, so we give a short sketch of the main steps. In the first step we show how the powers of $f(\alpha)$ can be reduced by using the functional equations. In the second step we consider $R\left(T^{k}(\alpha), f\left(T^{k}(\alpha)\right)\right)$ for a polynomial $R$ and construct by induction a polynomial $R_{k}$, with degrees and height depending only on the degrees and height of $R$ and on $d, \beta, d_{y}(\underline{P})$ and $k$, such that $\left|R_{k}(\alpha, f(\alpha))\right|$ has almost the same analytic bounds as $\left|R\left(T^{k}(\alpha), f\left(T^{k}(\alpha)\right)\right)\right|$. In the last step we use this polynomial $R_{k}$ to construct a suitable sequence of polynomials $Q_{k} \in \mathcal{O}_{\mathbb{K}}[y]$ satisfying the assumptions of Lemma 8 and prove Theorem 1 by Lemma 8 . 
For a real number $a$ we define $a_{+}:=\max \{a, 0\}=\frac{1}{2}(a+|a|)$.

Let $\mathbb{K}$ be an algebraic number field containing $\alpha$, the coefficients of $f_{1}, \ldots, f_{m}$ (cf. the assumption of Theorem 1 and Lemma 4) and the coefficients of the polynomials $a, P_{0,1}, \ldots, P_{n_{m}-1, m}$. Without loss of generality we can assume $a \in \mathcal{O}_{\mathbb{K}}[z]$ and $P_{0,1}, \ldots, P_{n_{m}-1, m} \in \mathcal{O}_{\mathbb{K}}[z, \underline{y}]$.

In what follows let $k \in \mathbb{N}$ be fixed. Under the conditions of Theorem 1 on $\alpha, d$ and $\underline{f}$ we put for abbreviation

$$
\tau_{\kappa}:=\alpha^{d^{\kappa}}, \quad \varphi_{i, \kappa}:=f_{i}\left(\alpha^{d^{\kappa}}\right) \quad \text { and } \quad \underline{\varphi}_{\kappa}:=\left(f_{1}\left(\alpha^{d^{\kappa}}\right), \ldots, f_{m}\left(\alpha^{d^{\kappa}}\right)\right) .
$$

For $j=1, \ldots, m$ let $P_{n_{j}, j}:=a$ and we define the following notations:

$$
\begin{aligned}
d_{z}(\underline{P}) & :=\max \left\{\operatorname{deg}_{z}\left(P_{0,1}\right), \ldots, \operatorname{deg}_{z}\left(P_{n_{m}, m}\right)\right\}, \\
d_{\underline{y}}(\underline{P}) & :=\max \left\{\operatorname{deg}_{\underline{y}}\left(P_{0,1}\right), \ldots, \operatorname{deg}_{\underline{y}}\left(P_{n_{m}, m}\right)\right\}, \\
L(\underline{P}) & :=\max \left\{L\left(P_{0,1}\right), \ldots, L\left(P_{n_{m}, m}\right)\right\} .
\end{aligned}
$$

Lemma 9. Suppose that $k \in \mathbb{N}$ and $\lambda \in \mathbb{N}_{0}$. Then for all $j=1, \ldots, m$ we have

$$
\left(a\left(\tau_{k-1}\right) f_{j}\left(\tau_{k}\right)\right)^{\lambda}=\sum_{i=0}^{n_{j}-1} P_{i, \lambda, j}^{(k)}\left(\tau_{k-1}, \underline{\varphi}_{k-1}\right)\left(a\left(\tau_{k-1}\right) f_{j}\left(\tau_{k}\right)\right)^{i}
$$

with polynomials $P_{i, \lambda, j}^{(k)} \in \mathcal{O}_{\mathbb{K}}[z, \underline{y}]$ satisfying

$$
\begin{aligned}
d_{z}\left(P_{i, \lambda, j}^{(k)}\right) & \leq(\lambda-i)_{+} d_{z}(\underline{P}), \\
d_{\underline{y}}\left(P_{i, \lambda, j}^{(k)}\right) & \leq(\lambda-i)_{+} d_{\underline{y}}(\underline{P}), \\
L\left(P_{i, \lambda, j}^{(k)}\right) & \leq 2^{\left(\lambda-n_{j}\right)_{+}} L(\underline{P})^{(\lambda-i)_{+}} .
\end{aligned}
$$

Proof. For $\lambda \in\left\{0, \ldots, n_{j}-1\right\}$ we choose $P_{i, \lambda, j}^{(k)}=\delta_{i, \lambda}$, where $\delta_{i, k}$ is the Kronecker symbol, and the assertions are obvious.

Let now $\lambda=n_{j}+l$ for $l \in \mathbb{N}_{0}$. We show the assertion by induction on $l$. This is obvious for $l=0$ because of $(2)$ and

$$
\left(a\left(\tau_{k-1}\right) f_{j}\left(\tau_{k}\right)\right)^{n_{j}}=\sum_{i=0}^{n_{j}-1} P_{i, j}\left(\tau_{k-1}, \underline{\varphi}_{k-1}\right) a\left(\tau_{k-1}\right)^{n_{j}-1-i}\left(a\left(\tau_{k-1}\right) f_{j}\left(\tau_{k}\right)\right)^{i},
$$

with $P_{i, n_{j}, j}^{(k)}(z, \underline{y}):=P_{i, j}(z, \underline{y}) a(z)^{n_{j}-1-i}$.

In the induction step the assertion follows from

$$
\begin{aligned}
\left(a\left(\tau_{k-1}\right) f_{j}\left(\tau_{k}\right)\right)^{n_{j}+l+1} & =\left(a\left(\tau_{k-1}\right) f_{j}\left(\tau_{k}\right)\right)^{n_{j}+l}\left(a\left(\tau_{k-1}\right) f_{j}\left(\tau_{k}\right)\right) \\
& =\sum_{i=0}^{n_{j}-1} P_{i, n_{j}+l, j}^{(k)}\left(\tau_{k-1}, \underline{\varphi}_{k-1}\right)\left(a\left(\tau_{k-1}\right) f_{j}\left(\tau_{k}\right)\right)^{i+1}
\end{aligned}
$$




$$
\begin{aligned}
= & \sum_{i=0}^{n_{j}-2} P_{i, n_{j}+l, j}^{(k)}\left(\tau_{k-1}, \underline{\varphi}_{k-1}\right)\left(a\left(\tau_{k-1}\right) f_{j}\left(\tau_{k}\right)\right)^{i+1} \\
& +P_{n_{j}-1, n_{j}+l, j}^{(k)}\left(\tau_{k-1}, \underline{\varphi}_{k-1}\right)\left(a\left(\tau_{k-1}\right) f_{j}\left(\tau_{k}\right)\right)^{n_{j}} \\
= & \sum_{i=0}^{n_{j}-2} P_{i, n_{j}+l, j}^{(k)}\left(\tau_{k-1}, \underline{\varphi}_{k-1}\right)\left(a\left(\tau_{k-1}\right) f_{j}\left(\tau_{k}\right)\right)^{i+1} \\
& +P_{n_{j}-1, n_{j}+l, j}^{(k)}\left(\tau_{k-1}, \underline{\varphi}_{k-1}\right) \\
& \times \sum_{i=0}^{n_{j}-1} P_{i, n_{j}, j}^{(k)}\left(\tau_{k-1}, \underline{\varphi}_{k-1}\right)\left(a\left(\tau_{k-1}\right) f_{j}\left(\tau_{k}\right)\right)^{i} \\
= & \sum_{i=0}^{n_{j}-1} P_{i, n_{j}+l+1, j}^{(k)}\left(\tau_{k-1}, \underline{\varphi}_{k-1}\right)\left(a\left(\tau_{k-1}\right) f_{j}\left(\tau_{k}\right)\right)^{i} .
\end{aligned}
$$

So we get

$$
P_{i, n_{j}+l+1, j}^{(k)}(z, \underline{y}):=P_{i-1, n_{j}+l, j}^{(k)}(z, \underline{y})+P_{n_{j}-1, n_{j}+l, j}^{(k)}(z, \underline{y}) P_{i, n_{j}, j}^{(k)}(z, \underline{y}),
$$

where $P_{-1, n_{j}+l, j}^{(k)}(z, \underline{y}):=0$.

By induction it follows that $P_{i, n_{j}+l+1, j}^{(k)} \in \mathcal{O}_{\mathbb{K}}[z, \underline{y}]$ and

$$
\begin{aligned}
& d_{z}\left(P_{i, n_{j}+l+1, j}^{(k)}\right) \leq\left(n_{j}+l+1-i\right) d_{z}(\underline{P}), \\
& d_{\underline{y}}\left(P_{i, n_{j}+l+1, j}^{(k)}\right) \leq\left(n_{j}+l+1-i\right) d_{\underline{y}}(\underline{P}), \\
& L\left(P_{i, n_{j}+l+1, j}^{(k)}\right) \leq 2^{l+1} L(\underline{P})^{n_{j}+l+1-i} .
\end{aligned}
$$

In the reduction step we replace $R\left(\tau_{k}, \underline{\varphi}_{k}\right)=: R_{0}\left(\tau_{k}, \varphi_{k}\right)$ for an arbitrary polynomial $R \in \mathcal{O}_{\mathbb{K}}[z, \underline{y}]$ inductively by $R_{l}\left(\tau_{k-l}, \underline{\varphi}_{k-l}\right)$ and finally get a polynomial $R_{k}$ with almost the same bounds for $\left|\overline{R_{k}}(\alpha, f(\alpha))\right|$, the degrees and the height of $R_{k}$ as $R_{0}$.

Lemma 10. Suppose $k \in \mathbb{N}$ and $R \in \mathcal{O}_{\mathbb{K}}[z, \underline{y}]$. Then there exists a polynomial

$$
R^{*}(z, \underline{u}, \underline{y}):=\sum_{\underline{\mu} \in M} R_{\underline{\mu}}^{*}(z, \underline{u}) \underline{y} \underline{\underline{\mu}} \in \mathcal{O}_{\mathbb{K}}[z, \underline{u}, \underline{y}]
$$

with $M:=\left\{0,1, \ldots, n_{1}-1\right\} \times \ldots \times\left\{0,1, \ldots, n_{k}-1\right\}$ and

$$
\begin{aligned}
d_{y_{j}}\left(R^{*}\right) & \leq n_{j}-1 \quad(j=1, \ldots, m), \\
d_{z}\left(R_{\underline{\mu}}^{*}\right) & \leq d d_{z}(R)+d_{z}(\underline{P}) d_{\underline{y}}(R), \\
d_{\underline{u}}\left(R_{\underline{\mu}}^{*}\right) & \leq d_{\underline{y}}(\underline{P}) d_{\underline{y}}(R), \\
L\left(R_{\underline{\mu}}^{*}\right) & \leq L(R) L(\underline{P})^{d_{\underline{y}}(R)} 2^{d_{\underline{y}}(R)}
\end{aligned}
$$


such that

$$
a\left(\tau_{k-1}\right)^{d_{y}(R)} R\left(\tau_{k}, \underline{\varphi}_{k}\right)=R^{*}\left(\tau_{k-1}, \underline{\varphi}_{k-1}, a\left(\tau_{k-1}\right) \underline{\varphi}_{k}\right) .
$$

Proof. From the representation

$$
R(z, \underline{y}):=\sum_{i=0}^{d_{z}(R)} \sum_{|\underline{j}| \leq d_{\underline{y}}(R)} R_{i, \underline{j}} z^{i} \underline{y} \underline{j}
$$

we get, by Lemma 9 ,

$$
\begin{aligned}
& a\left(\tau_{k-1}\right)^{d_{\underline{y}}(R)} R\left(\tau_{k}, \underline{\varphi}_{k}\right)=\sum_{i=0}^{d_{z}(R)} \sum_{|\underline{j}| \leq d_{\underline{y}}(R)} R_{i, \underline{j}} \tau_{k}^{i} a\left(\tau_{k-1}\right)^{d_{\underline{y}}(R)-|\underline{j}|}\left(a\left(\tau_{k-1}\right) \underline{\varphi}_{k}\right)^{\underline{j}} \\
& =\sum_{\underline{\mu} \in M} R_{\underline{\mu}}^{*}\left(\tau_{k-1}, \underline{\varphi}_{k-1}\right)\left(a\left(\tau_{k-1}\right) \underline{\varphi}_{k}\right)^{\underline{\mu}},
\end{aligned}
$$

where

$$
\begin{aligned}
& R_{\underline{\mu}}^{*}(z, \underline{u}) \\
& \quad:=\sum_{i=0}^{d_{z}(R)} \sum_{|\underline{j}| \leq d_{\underline{y}}(R)} R_{i, \underline{j}} z^{d^{i}} a(z)^{d_{y}(R)-|\underline{j}|} P_{\mu_{1}, j_{1}, 1}^{(k)}(z, \underline{u}) \cdot \ldots \cdot P_{\mu_{m}, j_{m}, m}^{(k)}(z, \underline{u}) .
\end{aligned}
$$

Now the bounds for the partial degrees $d_{y_{j}}$ are obvious. From Lemma 9 we get

$$
\begin{aligned}
d_{z}\left(R_{\underline{\mu}}^{*}\right) \leq & d d_{z}(R)+d_{z}(\underline{P}) d_{\underline{y}}(R) \\
& +d_{z}(\underline{P}) \max \left\{\sum_{i=1}^{\underline{m}}\left(j_{i}-\mu_{i}\right)_{+}-j_{i}:|\underline{j}| \leq d_{\underline{y}}(R)\right\} \\
\leq & d d_{z}(R)+d_{z}(\underline{P}) d_{\underline{y}}(R)
\end{aligned}
$$

and similarly we derive the upper bound for $d_{\underline{u}}$. The length can be bounded in an analogous way by

$$
\begin{aligned}
L\left(R_{\underline{\mu}}^{*}\right) & \leq L(R) 2^{\max \left\{\sum_{i=1}^{m}\left(j_{i}-n_{i}\right)_{+}:|\underline{j}| \leq d_{\underline{y}}(R)\right\}} L(\underline{P})^{d_{\underline{y}}(R)} \\
& \leq L(R) L(\underline{P})^{d_{\underline{y}}(R)} 2^{d_{\underline{y}}(R)} .
\end{aligned}
$$

Lemma 11. Suppose that $R^{*} \in \mathcal{O}_{\mathbb{K}}[z, \underline{u}, \underline{y}]$ is the polynomial in Lemma 10. Then there exist polynomials $U_{1}, \ldots, U_{\beta} \in \mathcal{O}_{\mathbb{K}}[z, \underline{u}]$ such that

$$
R^{* \beta}+U_{1} R^{* \beta-1}+\ldots+U_{\beta}=0
$$

at the point $\left(z_{0}, \underline{u}_{0}, \underline{y}_{0}\right):=\left(\tau_{k-1}, \underline{\varphi}_{k-1}, a\left(\tau_{k-1}\right) \underline{\varphi}_{k}\right)$ and

$$
\begin{aligned}
d_{z}\left(U_{l}\right) & \leq \beta d_{d_{z}}(R)+\beta d_{z}(\underline{P})\left(d_{\underline{y}}(R)+|\underline{n}|\right), \\
d_{\underline{u}}\left(U_{l}\right) & \leq \beta d_{\underline{y}}(\underline{P})\left(d_{\underline{y}}(R)+|\underline{n}|\right), \\
L\left(U_{l}\right) & \leq \exp \left(c_{13}\left(d_{z}(R)+d_{\underline{y}}(R)\right)\right) H(R)^{\beta} .
\end{aligned}
$$


Proof. With $R^{*}(z, \underline{u}, \underline{y}):=\sum_{\underline{\mu} \in M} R_{\underline{\mu}}^{*}(z, \underline{u}) \underline{y} \underline{\mu}$ as in Lemma 10 we put for $\underline{\nu} \in M$,

$$
\begin{aligned}
R^{*}\left(\tau_{k-1}, \underline{\varphi}_{k-1}, a\left(\tau_{k-1}\right) \underline{\varphi}_{k}\right)\left(a\left(\tau_{k-1}\right) \underline{\varphi}_{k}\right)^{\underline{\nu}} & \\
& =\sum_{\underline{\mu} \in M} R_{\underline{\mu}}^{*}\left(\tau_{k-1}, \underline{\varphi}_{k-1}\right)\left(a\left(\tau_{k-1}\right) \underline{\varphi}_{k}\right)^{\underline{\mu}}+\underline{\nu} \\
& =\sum_{\underline{\lambda} \in M} R_{\underline{\lambda}, \underline{\nu}}\left(\tau_{k-1}, \underline{\varphi}_{k-1}\right)\left(a\left(\tau_{k-1}\right) \underline{\varphi}_{k}\right)^{\underline{\lambda}},
\end{aligned}
$$

with (cf. Lemma 9)

$$
R_{\underline{\lambda}, \underline{\nu}}(z, \underline{u}):=\sum_{\underline{\mu} \in M} R_{\underline{\mu}}^{*}(z, \underline{u}) P_{\lambda_{1}, \mu_{1}+\nu_{1}, 1}^{(k)}(z, \underline{u}) \cdot \ldots \cdot P_{\lambda_{m}, \mu_{m}+\nu_{m}, m}^{(k)}(z, \underline{u}) .
$$

The degrees and length of $R_{\underline{\lambda}, \underline{\nu}}$ can be bounded by Lemmas 9 and 10:

$$
\begin{aligned}
d_{z}\left(R_{\underline{\lambda}, \underline{\nu}}\right) & \leq \max _{\underline{\mu} \in M}\left\{d_{z}\left(R_{\underline{\mu}}^{*}\right)+\sum_{j=1}^{m} d_{z}\left(P_{\lambda_{j}, \mu_{j}+\nu_{j}, j}^{(k)}\right)\right\} \\
& \leq d d_{z}(R)+d_{z}(\underline{P}) d_{\underline{y}}(R)+d_{z}(\underline{P}) \max _{\underline{\mu} \in M}\left\{\sum_{j=1}^{m}\left(\mu_{j}+\nu_{j}-\lambda_{j}\right)_{+}\right\} \\
& \leq d d_{z}(R)+d_{z}(\underline{P})\left(d_{\underline{y}}(R)+|\underline{n}|+|\underline{\nu}|-|\underline{\lambda}|\right) .
\end{aligned}
$$

Similarly

$$
\begin{aligned}
& d_{\underline{u}}\left(R_{\underline{\lambda}, \underline{\nu}}\right) \leq d_{\underline{y}}(\underline{P})\left(d_{\underline{y}}(R)+|\underline{n}|+|\underline{\nu}|-|\underline{\lambda}|\right), \\
& L\left(R_{\underline{\lambda}, \underline{\nu}}\right) \leq L(R) L(\underline{P})^{d_{\underline{y}}}(R)+|\underline{n}|+|\underline{\nu}|-|\underline{\lambda}| 2^{d_{\underline{y}}(R)+|\underline{\nu}|} \leq \gamma_{1} L(R) \gamma_{2}{ }^{d_{y}(R)},
\end{aligned}
$$

where the constants $\gamma_{1}, \gamma_{2} \in \mathbb{R}_{+}$depend only on $\underline{P}$ and $\underline{n}$.

Thus the system of $\beta$ linear equations with $\beta$ unknowns,

$$
\sum_{\underline{\lambda} \in M}\left\{R_{\underline{\lambda}, \underline{\nu}}\left(\tau_{k-1}, \underline{\varphi}_{k-1}\right)-\delta_{\underline{\lambda}, \underline{\nu}} R^{*}\left(\tau_{k-1}, \underline{\varphi}_{k-1}, a\left(\tau_{k-1}\right) \underline{\varphi}_{k}\right)\right\} \underline{\omega}_{\underline{\lambda}}=0,
$$

where

$$
\delta_{\underline{\lambda}, \underline{\nu}}:= \begin{cases}1 & \text { if } \underline{\lambda}=\underline{\nu} \\ 0 & \text { else }\end{cases}
$$

is the generalized Kronecker symbol, has for $\underline{\omega}:=\left(\underline{\omega}_{\underline{\lambda}}\right)_{\underline{\lambda}} \in M$ a nontrivial solution

$$
\underline{\omega}_{\underline{\lambda}}:=\left(a\left(\tau_{k-1}\right) \underline{\varphi}_{k}\right)^{\underline{\lambda}} .
$$

Hence the determinant of the matrix of coefficients must vanish at the point $\left(z_{0}, \underline{u}_{0}, \underline{y}_{0}\right):=\left(\tau_{k-1}, \underline{\varphi}_{k-1}, a\left(\tau_{k-1}\right) \underline{\varphi}_{k}\right)$, and the expansion of the determinant with respect to the powers of $R^{*}\left(\bar{\tau}_{k-1}, \underline{\varphi}_{k-1}, a\left(\tau_{k-1}\right) \underline{\varphi}_{k}\right)$ implies 


$$
0=\operatorname{det}\left(R_{\underline{\lambda}, \underline{\nu}}-\delta_{\underline{\lambda}, \underline{\nu}} R^{*}\right)_{\underline{\lambda}, \underline{\nu} \in M}= \pm\left(R^{* \beta}+U_{1} R^{* \beta-1}+\ldots+U_{\beta}\right)
$$

with polynomials $U_{l} \in \mathcal{O}_{\mathbb{K}}[z, \underline{u}]$.

Since the polynomials $U_{l}$ are sums of products of the form

$$
R_{\underline{\lambda}_{1}, \underline{\sigma}\left(\underline{\lambda}_{1}\right)} \cdot \ldots \cdot R_{\underline{\lambda}_{s}, \underline{\sigma}\left(\underline{\lambda}_{s}\right)},
$$

where $\underline{\lambda}_{1}, \ldots, \underline{\lambda}_{s} \in M$ are pairwise distinct and $\underline{\sigma}:=\left(\sigma_{1}, \ldots, \sigma_{m}\right)$ is a permutation of $\left\{0, \ldots, n_{1}-1\right\} \times \ldots \times\left\{0, \ldots, n_{m}-1\right\}$, for $l \in\{1, \ldots, \beta\}$ we get

$$
d_{\underline{u}}\left(U_{l}\right) \leq \max _{\underline{\sigma}}\left\{\sum_{\underline{\lambda} \in M} d_{\underline{u}}\left(R_{\underline{\lambda}, \underline{\sigma}(\underline{\lambda})}\right)\right\} \leq \beta d_{\underline{y}}(\underline{P})\left(d_{\underline{y}}(R)+|\underline{n}|\right)
$$

because

By analogy we obtain

$$
\sum_{\underline{\lambda} \in M}(|\underline{\lambda}|-|\underline{\sigma}(\underline{\lambda})|)=0
$$

$$
d_{z}\left(U_{l}\right) \leq \max _{\underline{\sigma}}\left\{\sum_{\underline{\lambda} \in M} d_{z}\left(R_{\underline{\lambda}, \underline{\sigma}(\underline{\lambda})}\right)\right\} \leq \beta d d_{z}(R)+\beta d_{z}(\underline{P})\left(d_{\underline{y}}(R)+|\underline{n}|\right) .
$$

The length of $U_{l}$ can be bounded by

$$
L\left(U_{l}\right) \leq \beta ! \max \left\{L\left(R_{\underline{\lambda}, \underline{\nu}}\right): \underline{\lambda}, \underline{\nu} \in M\right\}^{\beta},
$$

with

Lemma 11 is proved.

$$
L\left(R_{\underline{\lambda}, \underline{\nu}}\right) \leq \exp \left(c_{13}\left(d_{z}(R)+d_{\underline{y}}(R)\right)\right) H(R) .
$$

Now the necessary tools for the reduction step from $R_{0}$ to $R_{k}$ are complete, and we prove for $j=0, \ldots, k$ the existence of polynomials $R_{j} \in$ $\mathcal{O}_{\mathbb{K}}[z, \underline{y}]$ such that for $j=0$,

$$
\begin{gathered}
d_{z}\left(R_{0}\right):=d_{1,0}, \quad d_{\underline{y}}\left(R_{0}\right):=d_{2,0}, \quad \log H\left(R_{0}\right):=H_{0}, \\
\exp \left(-\psi_{1}(0)\right) \leq\left|R_{0}\left(\tau_{k}, \underline{\varphi}_{k}\right)\right| \leq \exp \left(-\psi_{2}(0)\right),
\end{gathered}
$$

and for $j \geq 1$ :

$$
\begin{aligned}
d_{\underline{y}}\left(R_{j}\right) & =: d_{2, j} \leq \beta d_{\underline{y}}(\underline{P})\left(d_{2, j-1}+|\underline{n}|\right), \\
d_{z}\left(R_{j}\right) & =: d_{1, j} \leq \beta d d_{1, j-1}+\beta d_{z}(\underline{P})\left(d_{2, j-1}+|\underline{n}|\right), \\
\log H\left(R_{j}\right) & =: H_{j} \leq \beta H_{j-1}+c_{14}\left(d_{1, j-1}+d_{2, j-1}\right) .
\end{aligned}
$$

Here the constant $c_{14}>0$ depends only on $\underline{f}$ and $\alpha$ and

$$
\exp \left(-\psi_{1}(j)\right) \leq\left|R_{j}\left(\tau_{k-j}, \underline{\varphi}_{k-j}\right)\right| \leq \exp \left(-\psi_{2}(j)\right) .
$$

The functions $\psi_{1}, \psi_{2}$ satisfy for $j \geq 1$ the following recurrence equalities:

$$
\begin{aligned}
& \psi_{1}(j):=\beta \psi_{1}(j-1)+\beta H_{j-1}+c_{15}\left(d_{1, j-1}+d^{k-j} d_{2, j-1}\right)+\log \beta, \\
& \psi_{2}(j):=\psi_{2}(j-1)-\beta H_{j-1}-c_{16}\left(d_{1, j-1}+d_{2, j-1}\right)-\log \beta
\end{aligned}
$$


provided that

$$
\psi_{2}(0) \geq c_{17} \beta^{k}\left(H_{0}+d^{k}\left(d_{1,0}+d_{2,0}\right)\right),
$$

where $c_{15}, c_{16}, c_{17} \in \mathbb{R}_{+}$are suitable constants depending only on $\underline{f}$ and $\alpha$.

The existence of the polynomials will be proved in the next section. First we will derive upper bounds for $d_{1, j}, d_{2, j}, H_{j}$ and $\psi_{1}(j)$ and a lower bound for $\psi_{2}(j)$.

Obviously (7) implies

$$
d_{2, j} \leq \gamma_{0}\left(\beta d_{\underline{y}}(\underline{P})\right)^{j}\left(d_{2,0}+|\underline{n}|\right) \leq c_{18}\left(\beta d_{\underline{y}}(\underline{P})\right)^{j} d_{2,0},
$$

and for $d_{1, j}$ we get inductively (note that $d>d_{\underline{y}}(\underline{P})$ by the condition of Theorem 1)

$$
d_{1, j} \leq(\beta d)^{j} d_{1,0}+\beta d_{z}(\underline{P}) \sum_{i=0}^{j-1}(\beta d)^{i}\left(d_{2, j-i-1}+|\underline{n}|\right) \leq c_{19}(\beta d)^{j}\left(d_{1,0}+d_{2,0}\right) .
$$

For $H_{j}$, the logarithm of the height of $R_{j}$, we get in a similar way

$$
H_{j} \leq \beta^{j} H_{0}+\gamma_{1} \sum_{i=0}^{j-1} \beta^{i}\left(d_{1, j-i-1}+d_{2, j-i-1}\right) \leq \beta^{j} H_{0}+c_{20}\left(d_{1,0}+d_{2,0}\right)(\beta d)^{j} .
$$

Now we can easily deduce from (11) and the above estimates that

(14) $\psi_{1}(k)=\beta^{k} \psi_{1}(0)$

$$
\begin{aligned}
& +\sum_{i=0}^{k-1} \beta^{i}\left\{\beta H_{k-i-1}+c_{15}\left(d_{1, k-i-1}+d^{i} d_{2, k-i-1}\right)+\log \beta\right\} \\
\leq & \beta^{k} \psi_{1}(0)+k \beta^{k} H_{0}+c_{21}(\beta d)^{k}\left(d_{1,0}+d_{2,0}\right) .
\end{aligned}
$$

In a similar way (cf. (13)) we can derive a lower bound for $\psi_{2}(k)$ :

$$
\begin{aligned}
\psi_{2}(k) & =\psi_{2}(0)-\sum_{i=0}^{k-1}\left\{\beta H_{k-i-1}+c_{16}\left(d_{1, k-i-1}+d_{2, k-i-1}\right)+\log \beta\right\} \\
& \geq \psi_{2}(0)-c_{22} \beta^{k}\left(H_{0}+d^{k}\left(d_{1,0}+d_{2,0}\right)\right) .
\end{aligned}
$$

Now we prove by induction on $j=0, \ldots, k$ the existence of a sequence of polynomials $R_{j} \in \mathcal{O}_{\mathbb{K}}[z, y]$ satisfying the conditions (6)-(10). For $j=0$, this is a consequence of Lemmas 5 and 6 with $R_{0}:=R$ and

$$
\begin{aligned}
& d_{1,0}, d_{2,0} \leq N, \quad H_{0} \leq c_{3} N^{(m+1) L}, \\
& \psi_{1}(0):=c_{10} \nu d^{k}, \quad \psi_{2}(0):=c_{11} \nu d^{k}
\end{aligned}
$$

provided that $d^{k} \geq c_{9} \nu^{L}$ for a suitable constant $c_{9}>0$. Now suppose that the assertions are true for $j-1(j \in\{1, \ldots, k\})$. We apply Lemmas 10 and 11 with $R$ replaced by $R_{j-1}$. This yields the existence of polynomials 
$U_{1}, \ldots, U_{\beta} \in \mathcal{O}_{\mathbb{K}}[z, \underline{u}]$ with

$$
\begin{aligned}
d_{z}\left(U_{l}\right) & \leq \beta d d_{1, j-1}+\beta d_{z}(\underline{P})\left(d_{2, j-1}+|\underline{n}|\right), \\
d_{\underline{u}}\left(U_{l}\right) & \leq \beta d_{\underline{y}}(\underline{P})\left(d_{2, j-1}+|\underline{n}|\right), \\
\log H\left(U_{l}\right) & \leq \gamma_{1}\left(d_{1, j-1}+d_{2, j-1}\right)+\beta H_{j-1}
\end{aligned}
$$

for $l=1, \ldots, \beta$ such that

$$
R_{j-1}^{* \beta}+U_{1} R_{j-1}^{* \beta-1}+\ldots+U_{\beta}=0
$$

for $\left(z_{0}, \underline{u}_{0}, \underline{y}_{0}\right):=\left(\tau_{k-j}, \underline{\varphi}_{k-j}, a\left(\tau_{k-j}\right) \underline{\varphi}_{k-(j-1)}\right)$. Here $R_{j-1}^{*} \in \mathcal{O}_{\mathbb{K}}[z, \underline{u}, \underline{y}]$ is defined analogously to Lemma 10 by

$$
\begin{aligned}
a\left(\tau_{k-j}\right)^{d_{2, j-1}} R_{j-1}\left(\tau_{k-(j-1)}, \underline{\varphi}_{k-(j-1)}\right) & \\
& =R_{j-1}^{*}\left(\tau_{k-j}, \underline{\varphi}_{k-j}, a\left(\tau_{k-j}\right) \underline{\varphi}_{k-(j-1)}\right) .
\end{aligned}
$$

The induction hypothesis together with the fact that $-\gamma_{2} d^{k} \leq \log \left|a\left(\tau_{k}\right)\right| \leq$ $\gamma_{3}$ for $k \in \mathbb{N}_{0}$, implies

$$
\begin{aligned}
-\psi_{1}(j-1)-\gamma_{4} d^{k-j} d_{2, j-1} & \leq \log \left|R_{j-1}^{*}\left(\tau_{k-j}, \underline{\varphi}_{k-j}, a\left(\tau_{k-j}\right) \underline{\varphi}_{k-(j-1)}\right)\right| \\
& \leq-\psi_{2}(j-1)+\gamma_{5} d_{2, j-1} .
\end{aligned}
$$

For $l=1, \ldots, \beta$ we obtain by a standard estimate together with Lemma 11,

$$
\begin{aligned}
\left|U_{l}\left(\tau_{k-j}, \underline{\varphi}_{k-j}\right)\right| & \leq L\left(U_{l}\right) \max \left\{1,\left|\tau_{k-j}\right|,\left|\varphi_{1, k-j}\right|, \ldots,\left|\varphi_{m, k-j}\right|\right\}^{d_{z}\left(U_{l}\right)+d_{\underline{u}}\left(U_{l}\right)} \\
& \leq \exp \left(\beta H_{j-1}+\gamma_{6}\left(d_{1, j-1}+d_{2, j-1}\right)\right),
\end{aligned}
$$

where the constant $\gamma_{6} \in \mathbb{R}_{+}$depends only on $f$ and $\alpha$.

By (13) and (16) we see that

$$
\psi_{2}(j-1)-\left(\beta H_{j-1}+\gamma_{7}\left(d_{1, j-1}+d_{2, j-1}\right)+\log \beta\right)>0
$$

and by Lemma 7 we get the existence of $l_{0} \in\{1, \ldots, \beta\}$ such that

$$
\begin{aligned}
\log \left|U_{l_{0}}\left(\tau_{k-j}, \underline{\varphi}_{k-j}\right)\right| \leq & -\psi_{2}(j-1)+\gamma_{8} d_{2, j-1}+\beta H_{j-1} \\
& +\gamma_{9}\left(d_{1, j-1}+d_{2, j-1}\right)+\log \beta \\
\leq & -\psi_{2}(j-1)+\beta H_{j-1}+c_{16}\left(d_{1, j-1}+d_{2, j-1}\right)+\log \beta \\
= & -\psi_{2}(j)
\end{aligned}
$$

and

$$
\begin{aligned}
& \log \left|U_{l_{0}}\left(\tau_{k-j}, \underline{\varphi}_{k-j}\right)\right| \\
& \geq-\beta \psi_{1}(j-1)-\gamma_{10} \beta d^{k-j} d_{2, j-1}-\beta H_{j-1} \\
&-\gamma_{11}\left(d_{1, j-1}+d_{2, j-1}\right)-\log \beta \\
& \geq-\beta \psi_{1}(j-1)-\beta H_{j-1}-c_{15}\left(d_{1, j-1}+\beta d^{k-j} d_{2, j-1}\right)-\log \beta \\
&=-\psi_{1}(j) .
\end{aligned}
$$


Thus we put $R_{j}(z, \underline{y}):=U_{l_{0}}(z, \underline{y}) \in \mathcal{O}_{\mathbb{K}}[z, \underline{y}]$ and see that (6)-(10) are proved for the polynomial $R_{j}$.

4. Proof of Theorem 1. Now the necessary tools for the proof of Theorem 1 are complete. From the preceding section with $j=k$ we know that for $k, N \in \mathbb{N}$ sufficiently large with

$$
\begin{aligned}
d^{k} & \geq c_{9} \nu^{L}, \\
\nu d^{k} & \geq c_{23} \beta^{k}\left(N^{(1+m) L}+d^{k} N\right)
\end{aligned}
$$

for sufficiently large constants $c_{9}, c_{23}>0$, there exist polynomials $R_{k} \in$ $\mathcal{O}_{\mathbb{K}}[z, \underline{y}]$ with

$$
\begin{aligned}
d_{z}\left(R_{k}\right) & \leq c_{24}(\beta d)^{k} N, \\
d_{\underline{y}}\left(R_{k}\right) & \leq c_{18}\left(\beta d_{\underline{y}}(\underline{P})\right)^{k} N, \\
\log H\left(R_{k}\right) & \leq c_{25}(\beta d)^{k} N, \\
-c_{26}(\beta d)^{k} \nu & \leq \log |R(\alpha, \underline{f}(\alpha))| \leq-c_{27} d^{k} \nu .
\end{aligned}
$$

The estimates for the degrees (19) and (20) are obvious from (16) and the above estimates. The upper bound for the height (21) of $R_{k}$ and a lower bound for the right-hand side of (22) could be derived from (18) and (15).

With (14) and (16) it follows from (18) that

$$
\psi_{1}(k) \leq \gamma_{1} \beta^{k} d^{k} \nu+\gamma_{2} k \beta^{k}\left(N^{(1+m) L}+d^{k} N\right) \leq \gamma_{1} \beta^{k} d^{k} \nu+\gamma_{3} k d^{k} \nu
$$

and this gives the left-hand inequality of (22); note that $\beta \geq 2$. by

In order to use Lemma 8 we define the polynomials $\left(Q_{k}\right)_{k_{0} \leq k \leq k_{1}} \in \mathcal{O}_{\mathbb{K}}[\underline{y}]$

$$
Q_{k}(\underline{y}):=D^{d_{z}\left(R_{k}\right)} R_{k}(\alpha, \underline{y}),
$$

where $D \in \mathbb{N}$ is a denominator of $\alpha$.

Because of (18) and (19) and the condition $d_{y}(\underline{P})<d$ we obtain, for $k \in \mathbb{N}$,

$$
\begin{aligned}
d_{\underline{y}}\left(Q_{k}\right) & \leq c_{18}\left(\beta d_{\underline{y}}(\underline{P})\right)^{k} N, \\
\log H\left(Q_{k}\right) & \leq c_{28}(\beta d)^{k} N, \\
\log \left|Q_{k}(\underline{f}(\alpha))\right| & \leq-c_{29} d^{k} \nu+c_{30}(\beta d)^{k} N \leq-c_{31} \nu d^{k}, \\
\log \left|Q_{k}(\underline{f}(\alpha))\right| & \geq-c_{32} \nu(\beta d)^{k} .
\end{aligned}
$$

Now for $N \in \mathbb{N}$ we define a number $M \geq N$ by $\nu:=c_{4} M^{m+1}$ and for positive integers $k_{0} \leq k \leq k_{1}$, where $k_{0}<k_{1}$ will be specified later, we 
define the following functions:

$$
\begin{gathered}
\Phi_{1}:=c_{18}\left(\beta d_{\underline{y}}(\underline{P})\right)^{k_{1}} M, \quad \Phi_{2}:=c_{28}(\beta d)^{k_{1}} M, \\
\psi_{1}(k):=c_{32} \nu(\beta d)^{k}, \quad \psi_{2}(k):=c_{31} \nu d^{k}, \\
\Lambda(k):=\frac{\psi_{1}(k+1)}{\psi_{2}(k)}=\frac{c_{32} d \beta}{c_{31}} \beta^{k} .
\end{gathered}
$$

With a sufficiently large constant $\gamma_{4} \in \mathbb{R}_{+}$we define, for $\nu=c_{4} M^{1+m}$,

$$
k_{0}:=\left[\frac{(1+m) L \log M}{\log d}+\gamma_{4}\right] .
$$

Then (17) and condition (i) of Lemma 8 are obviously fulfilled for all $k \geq k_{0}$.

For $M \geq N$ large enough we have to find a positive integer $k_{1}=k_{1}(M)$ $>k_{0}$ such that the inequalities (ii) and (iii) of Lemma 8 are satisfied, where the condition (iii) is equivalent to the following two inequalities:

$$
\begin{gathered}
\left(\frac{d}{\beta^{2\left(m_{0}-1\right)} d_{\underline{y}}(\underline{P})^{m_{0}-1}}\right)^{k_{1}} \geq c_{33} M^{m_{0}-1}(d \beta)^{k_{0}}, \\
M^{m+1-m_{0}} \geq c_{34}\left(\beta^{2\left(m_{0}-1\right)+1} d_{\underline{y}}(\underline{P})^{m_{0}-1}\right)^{k_{1}}
\end{gathered}
$$

with ineffective constants $c_{33}, c_{34} \in \mathbb{R}_{+}$.

REMARK. In the inequality $(24)$ we see that the condition $d>d_{\underline{y}}(\underline{P})$ is necessary to obtain nontrivial results.

Since

$$
m_{0}<\frac{m-\sigma L(m+1)(1+\sigma)}{\sigma+1+(L(m+1)(1+\sigma)+m)\left(2 \sigma+\log d_{\underline{y}}(\underline{P}) / \log d\right)}+1
$$

with $\sigma:=\log \beta / \log d$, the inequality

$$
\begin{aligned}
\left(\left(m_{0}-1\right) \log \left(\beta^{2} d_{\underline{y}}(\underline{P})\right)+\log \beta\right)\left(\left(m_{0}-1\right)+L(m+1)(1+\sigma)\right) \\
<\left(m+1-m_{0}\right)\left(\log d-\left(m_{0}-1\right) \log \left(\beta^{2} d_{\underline{y}}(\underline{P})\right)\right)
\end{aligned}
$$

holds. So we can find $\gamma \in \mathbb{R}_{+}$satisfying

$$
\begin{aligned}
m+1-m_{0} & >\gamma\left(\left(m_{0}-1\right) \log \left(\beta^{2} d_{\underline{y}}(\underline{P})\right)+\log \beta\right), \\
\left(m_{0}-1\right)+L(m+1)(1+\sigma) & <\gamma\left(\log d-\left(m_{0}-1\right) \log \left(\beta^{2} d_{\underline{y}}(\underline{P})\right)\right) .
\end{aligned}
$$

Now we choose $N \in \mathbb{N}$ and thereby $M$ large enough, define $k_{1}$ by $k_{1}:=$ $[\gamma \log M]$ and show that the conditions $k_{0}<k_{1}$ and (18) are fulfilled.

Without loss of generality, we may assume that $m_{0} \geq 1$ and see that

$$
\gamma>\frac{\left(m_{0}-1\right)+L(m+1)(1+\sigma)}{\log d-\left(m_{0}-1\right) \log \left(\beta^{2} d_{\underline{y}}(\underline{P})\right)} \geq \frac{L(m+1)}{\log d},
$$

which shows $k_{0}<k_{1}$. 
To see that (18) is fulfilled, we show that $\nu d^{k} \geq \gamma_{5} \beta^{k} d^{k} M$ and $\nu d^{k} \geq$ $\gamma_{6} \beta^{k} M^{L(m+1)}$ is valid for $k_{0} \leq k \leq k_{1}$.

As $m_{0} \geq 1$ we get

$$
\gamma<\frac{m+1-m_{0}}{\left(m_{0}-1\right) \log \left(\beta^{2} d_{y}(\underline{P})\right)+\log \beta} \leq \frac{m}{\log \beta},
$$

and the inequality $\nu d^{k} \geq \gamma_{5} \beta^{k} d^{k} M$ is obvious.

A similar argument leads to $\nu d^{k} \geq \gamma_{6} \beta^{k} M^{L(m+1)} \geq 1$. From the condition $d>\beta^{L}$ we obtain, for $k \geq k_{0}$,

$$
k(\log d-\log \beta)>\frac{(1+m) L}{\log d} \log M(\log d-\log \beta)>(L-1)(m+1) \log M,
$$

hence

$$
\left(\frac{d}{\beta}\right)^{k} \geq\left(\frac{d}{\beta}\right)^{k_{0}} \geq \gamma_{7} M^{(L-1)(m+1)} .
$$

Now we can finish the proof of Theorem 1. We have shown that the conditions (17) and (18) are satisfied with this choice of parameters, if $N \in \mathbb{N}$ is large enough with respect to a constant depending only on $\alpha$ and $f$. We get

$$
\begin{aligned}
k_{1}\left(\log d-\left(m_{0}-1\right) \log \left(\beta^{2} d_{\underline{y}}(\underline{P})\right)\right) & \\
& \geq\left(\left(m_{0}-1\right)+L(m+1)(1+\sigma)\right) \log M+c, \\
\left(m+1-m_{0}\right) \log M & \geq k_{1}\left(\left(m_{0}-1\right) \log \left(\beta^{2} d_{\underline{y}}(\underline{P})\right)+\log \beta\right)+c,
\end{aligned}
$$

for a suitable constant $c>0$. This implies the inequalities (24) and (25), hence the condition (iii) of Lemma 8 and thereby the assertion of Theorem 1.

\section{Proof of the algebraic independence of the functions consid-} ered in Corollary 3. Let

$$
f_{n}(z):=\prod_{j=0}^{\infty}\left(1-z^{d^{j}}\right)^{n^{j}} .
$$

By induction on $k$ we prove that for $\left\{i_{1}, \ldots, i_{k}\right\} \subset\{1, \ldots, m\}$, where $i_{1}, \ldots$ $\ldots, i_{k}$ are pairwise distinct, the functions $f_{n_{i_{1}}}, \ldots, f_{n_{i_{k}}}$ are algebraically independent over $\mathbb{C}(z)$. We follow the proof of Proposition 6 in [10].

For abbreviation we put for $j=1, \ldots, k$ and a positive integer $\nu \in \mathbb{N}$

$$
f_{n_{i_{j}}}(z):=\varphi_{j} \quad \text { and } \quad f_{n_{i_{j}}}\left(z^{d^{\nu}}\right):=\varphi_{j}^{(\nu)} .
$$

Assume that $\varphi_{1}$ is algebraic over $\mathbb{C}(z)$. Then by Theorem 1.3 of [11] it is a rational function. Let $\varphi_{1}=a(z) / b(z)$, where $a(z)$ and $b(z)$ are relatively 
prime polynomials. By the functional equation we obtain

$$
a(z) b\left(z^{d}\right)^{n_{i_{1}}}=(1-z) a\left(z^{d}\right)^{n_{i_{1}}} b(z) .
$$

Since $a$ and $b$ are relatively prime polynomials, we get $a\left(z^{d}\right)^{n_{i_{1}}} \mid a(z)$, hence $a \in \mathbb{C}^{*}$ and

$$
(1-z) a^{n_{i_{1}}-1} b(z)=b\left(z^{d}\right)^{n_{i_{1}}} .
$$

If $d n_{i_{1}}>2$ or $\operatorname{deg} b \geq 2$, we get a contradiction by comparing the degrees. In the remaining case it is enough to assume $b(z)=\alpha z+\beta$; then by considering the equation $(1-z) b(z)=b\left(z^{2}\right)$ we see $\alpha=\beta=0$ and again we obtain a contradiction.

Assume now that the assertion is true for $k-1$, but $\left\{f_{n_{i_{1}}}(z), \ldots, f_{n_{i_{k}}}(z)\right\}$ $=:\left\{\varphi_{1}, \ldots, \varphi_{k}\right\}$ are algebraically dependent over $\mathbb{C}(z)$. By $D^{(\nu)}$ and $D_{\kappa}^{(\nu)}$ we denote the degrees of the following field extensions:

$$
\begin{aligned}
D^{(\nu)} & :=\left[\mathbb{C}(z)\left(\varphi_{1}^{(\nu)}, \ldots, \varphi_{k}^{(\nu)}\right): \mathbb{C}(z)\left(\varphi_{1}, \ldots, \varphi_{k}\right)\right], \\
D_{\kappa}^{(\nu)} & :=\left[\mathbb{C}(z)\left(\varphi_{1}^{(\nu)}, \ldots, \widehat{\varphi_{\kappa}^{(\nu)}}, \ldots, \varphi_{k}^{(\nu)}\right): \mathbb{C}(z)\left(\varphi_{1}, \ldots, \widehat{\varphi}_{\kappa}, \ldots, \varphi_{k}\right)\right],
\end{aligned}
$$

where $\left(\varphi_{1}, \ldots, \widehat{\varphi}_{\kappa}, \ldots, \varphi_{k}\right):=\left(\varphi_{1}, \ldots, \varphi_{\kappa-1}, \varphi_{\kappa+1}, \ldots, \varphi_{k}\right)$.

In a first step we show that for arbitrary positive integers $n$ and $\nu$,

$$
\left[\mathbb{C}(z)\left(f_{n}\left(z^{d^{\nu}}\right)\right): \mathbb{C}(z)\left(f_{n}(z)\right)\right]=n^{\nu} ;
$$

but this is trivial by induction, since the polynomial $P(y):=(1-z) y^{n}-$ $f_{n}(z) \in \mathbb{C}\left(z, f_{n}(z)\right)[y]$ is irreducible. (Note that $f_{n}(z)$ is not an algebraic function.)

Now we are able to prove

$$
D_{\kappa}^{(\nu)}=\left(\prod_{\lambda=1, \lambda \neq \kappa}^{k} n_{i_{\lambda}}\right)^{\nu}=\left(\prod_{\lambda=1}^{k} n_{i_{\lambda}}\right)^{\nu} n_{i_{\kappa}}^{-\nu} .
$$

We prove this formula for simplicity just for $k=\kappa=3$, but the general case follows similarly.

Since by assumption $\varphi_{1}$ and $\varphi_{2}$ are algebraically independent, we see by the functional equation that $\varphi_{1}^{(\nu)}$ and $\varphi_{2}^{(\nu)}$ are also algebraically independent. Hence $\mathbb{C}(z)\left(\varphi_{1}^{(\nu)}\right)$ and $\mathbb{C}(z)\left(\varphi_{2}^{(\nu)}\right)$ are regular field extensions (cf. Weil [18]), which are linearly disjoint by [18, Theorem I.6]. The assumption now follows from [18, Proposition I.14].

Let $d_{\kappa}$ be the degree of $\varphi_{\kappa}$ over $\mathbb{C}(z)\left(\varphi_{1}, \ldots, \widehat{\varphi}_{\kappa}, \ldots, \varphi_{k}\right)$, then we get $d_{\kappa}^{(\nu)} \leq d_{\kappa}$, where $d_{\kappa}^{(\nu)}$ denotes the degree of $\varphi_{\kappa}^{(\nu)}$ over $\mathbb{C}(z)\left(\varphi_{1}^{(\nu)}, \ldots, \widehat{\varphi_{\kappa}^{(\nu)}}, \ldots\right.$ $\left.\ldots, \varphi_{k}^{(\nu)}\right)$. Finally we obtain by a standard formula

$$
D^{(\nu)} d_{\kappa}=d_{\kappa}^{(\nu)} D_{\kappa}^{(\nu)} .
$$


Let $\mu, \kappa \in\{1, \ldots, k\}$ and $n_{i_{\mu}}<n_{i_{\kappa}}$. By the above formulas we get

$$
\left(\frac{n_{i_{\kappa}}}{n_{i_{\mu}}}\right)^{\nu}=\frac{D_{\mu}^{(\nu)}}{D_{\kappa}^{(\nu)}}=\frac{d_{\mu}}{d_{\mu}^{(\nu)}} \cdot \frac{d_{\kappa}^{(\nu)}}{d_{\kappa}} \leq d_{\mu} .
$$

Since $n_{i_{\mu}}<n_{i_{\kappa}}$, this is a contradiction as $\nu$ tends to infinity. Thus the algebraic independence of the functions $f_{n_{1}}, \ldots, f_{n_{m}}$ is proved.

Acknowledgements. The result of this paper is part of the author's thesis written under the direction of Professor P. Bundschuh. The author wants to express his gratitude to him for his encouragement and helpful comments.

\section{References}

[1] P.-G. Becker, Transcendence of the values of functions satisfying generalized Mahler type functional equations, J. Reine Angew. Math. 440 (1993), 111-128.

[2] V. G. Chirskiŭ, On the algebraic independence of the values of functions satisfying systems of functional equations, Proc. Steklov Inst. Math. 218 (1997), 433-438.

[3] J. H. Loxton and A. J. van der Poorten, Transcendence and algebraic independence by a method of Mahler, in: Transcendence Theory-Advances and Applications, A. Baker and D. W. Masser (eds.), Academic Press, New York, 1977, 211-226.

[4] K. Mahler, Arithmetische Eigenschaften der Lösungen einer Klasse von Funktionalgleichungen, Math. Ann. 101 (1929), 342-366.

[5] - , Arithmetische Eigenschaften einer Klasse transzendental-transzendenter Funktionen, Math. Z. 32 (1930), 545-585.

[6] —, Über das Verschwinden von Potenzreihen mehrerer Veränderlichen in speziellen Punktfolgen, Math. Ann. 103 (1930), 573-587.

[7] —, Remarks on a paper by W. Schwarz, J. Number Theory 1 (1969), 512-521.

[8] K. Nishioka, On a problem of Mahler for transcendency of function values, J. Austral. Math. Soc. Ser. A 33 (1982), 386-393.

[9] - On a problem of Mahler for transcendency of function values II, Tsukuba J. Math. 7 (1983), 265-279.

[10] -, New approach in Mahler's method, J. Reine Angew. Math. 407 (1990), 202-219.

[11] - Mahler Functions and Transcendence, Lecture Notes in Math. 1631, Springer, 1996.

[12] T. Schneider, Einführung in die transzendenten Zahlen, Springer, Berlin, 1957.

[13] T. Töpfer, An axiomatization of Nesterenko's method and applications on Mahler functions, J. Number Theory 49 (1994), 1-26.

[14] - Algebraic independence of the values of generalized Mahler functions, Acta Arith. 70 (1995), 161-181.

[15] - Simultaneous approximation measures for functions satisfying generalized functional equations of Mahler type, Abh. Math. Sem. Univ. Hamburg 66 (1996), 177201. 
[16] M. Waldschmidt, Algebraic independence of transcendental numbers: a survey, in: Proceedings of the Fifth Conference of the Canadian Number Theory Association; Monograph on Number Theory, Indian National Science Academy, R. P. Bambah et al. (eds.), to appear; http://www.math.jussieu.fr/ ${ }^{\sim}$ miw, 1998.

[17] N. C. Wass, Algebraic independence of the values at algebraic points of a class of functions considered by Mahler, Dissertationes Math. 303 (1990).

[18] A. Weil, Foundations of Algebraic Geometry, Colloq. Publ. 29, Amer. Math. Soc., 1962 .

Lechenicher Str. 18

D-50937 Köln, Germany

Received on 5.10 .1998

and in revised form on 1.3.1999 and 30.8.1999 